\title{
High-dose radiotherapy in trimodality treatment of Pancoast tumors results in high pathologic complete response rates and excellent long-term survival
}

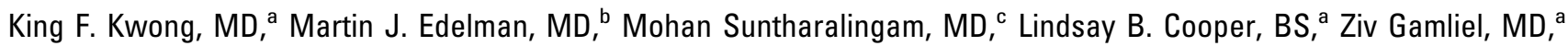
Whitney Burrows, MD, ${ }^{a}$ Petr Hausner, MD, PhD, ${ }^{b}$ L. Austin Doyle, MD, and Mark J. Krasna, MD ${ }^{\mathrm{a}}$

From the Divisions of Thoracic Surgery ${ }^{\mathrm{a}}$ and Medical Oncology ${ }^{\mathrm{b}}$ and the Department of Radiation Oncology, ${ }^{\mathrm{c}}$ University of Maryland School of Medicine and Greenebaum Cancer Center, Baltimore, Md.

Read at the Eighty-fourth Annual Meeting of The American Association for Thoracic Surgery, Toronto, Ontario, Canada, April 25-28, 2004.

Received for publication May 13, 2004; revisions received Dec 7, 2004; accepted for publication Dec 17, 2004.

Address for reprints: Mark J. Krasna, MD, Division of Thoracic Surgery, University of Maryland School of Medicine, 22 S. Greene St, Room N4E35, Baltimore, MD 21201 (E-mail: mkrasna@smail.umaryland.edu).

J Thorac Cardiovasc Surg 2005;129:1250-7

$0022-5223 / \$ 30.00$

Copyright (c) 2005 by The American Association for Thoracic Surgery

doi:10.1016/j.jtcvs.2004.12.050
Objective: We sought to study the clinical characteristics and outcomes of patients treated with a surgery-inclusive multimodality approach for Pancoast tumors.

Methods: Clinical records of patients with Pancoast lung cancer who were enrolled for multimodality treatment between 1993 and 2003 at our institution were reviewed retrospectively.

Results: Thirty-six patients completed neodjuvant chemoradiation followed by en bloc surgical resection, whereas one patient received high-dose radiation alone followed by surgical intervention. There were 22 men and 15 women. Thirty-four lobectomies and 3 pneumonectomies were performed. Pretreatment non-small cell lung cancer stages were IIB, IIIA, IIIB, and IV (presenting with solitary brain metastasis) in 18, 8, 6, and 5 cases, respectively. R0 resection was achieved in 36 $(97.3 \%)$ patients. Operative mortality was $2.7 \%(\mathrm{n}=1)$. High-dose radiotherapy was successfully tolerated in all but 1 patient. Mean total radiation dose was 56.9 Gy. Pathologic complete response was found in $40.5 \%(\mathrm{n}=15)$ of patients. Recurrences were found in 50\% $(\mathrm{n}=18)$ of patients. Brain metastasis was the most common recurrence $(n=9)$, followed by other distant recurrences $(n=4)$ and local recurrences $(\mathrm{n}=5)$. Median survival time for the group is 2.6 years, and median survival time (pathologic complete response) is 7.8 years. It is noteworthy that median survival time of patients with positive pretreatment lymph nodes (12 patients) was not reached.

Conclusions: Surgical resection of Pancoast tumors after neoadjuvant high-dose radiation and chemotherapy can be safely performed. High-dose radiation in trimodality treatment is well tolerated and might be beneficial. Similar to other studies, late central nervous system relapse is problematic and indicates a need for assessing the role of prophylactic cranial irradiation in this disease.

$\mathrm{T}$ The eponym Pancoast tumor derives from the noted Philadelphia radiologist Dr. Henry K. Pancoast's description of these superior sulcus tumors in his classic article published in $1924 .{ }^{1}$ Currently accepted criteria characterizing Pancoast tumors emphasize features such as location at the superior sulcus and involvement of structures of the apical chest wall above the level of the second rib. ${ }^{2}$ Symptomatology in patients with Pancoast tumors might be quite variable, depending on which apical structures are affected by local tumor abutment or invasion. Although Pancoast tumors comprise less than 5\% of all lung cancers, the significant morbidity of untreated tumors and the challenges inherent in effectively treating these cancers because of their anatomic location have provided tremendous incentive to clinicians to seek improved outcomes in this select patient population.

In the last 40 years, the treatment of Pancoast tumors has centered on a bimodality regimen consisting of preoperative external beam radiotherapy followed 
by surgical resection, as serendipitously discovered and subsequently described in 1961 by Shaw and Paulson.,4 The recent addition of chemotherapy to preoperative radiation is motivated by the rationale of improving resectability rates and preventing systemic disease. ${ }^{5-8}$ However, the optimum neoadjuvant radiation dosage or chemotherapy regimen remains to be determined. In this article we examined our center's experience with Pancoast tumors in the last decade to assess the effects of high-dose radiation instituted in a neoadjuvant setting with combination chemotherapy on clinical outcomes.

\section{Methods}

We retrospectively analyzed all patients with Pancoast tumors treated with a multimodality approach at our institution from 1993 through 2003. Tumors involving the chest wall only and deemed resectable by means of surgical intervention alone were not included in this study. This study was approved by the Institutional Review Board of the University of Maryland (UMB Protocol H23742).

\section{Pancoast Tumor Identification}

Patients demonstrating radiographic and clinical signs of superior sulcus tumors were included. Preoperative pathologic diagnosis was made with computed tomography (CT)-guided fine-needle aspiration biopsy. Preresection lymph node stage was determined by means of either transbronchial biopsy or mediastinoscopy in all patients. Other radiologic examinations, such as brain CT or magnetic resonance imaging and positron emission tomography were obtained as clinically warranted.

\section{Induction Therapy Regimen}

All patients underwent preoperative high-dose radiotherapy with an intent-to-treat approach. Those patients receiving chemotherapy in combination with radiotherapy began both treatments within 24 hours of each other. Weekly platinum-based combination chemotherapy was used in the majority of patients. Three-dimensional conformal radiation therapy was administered as a large-field 45-Gy dose to the primary tumor and mediastinum, followed by a small-field boost to the primary tumor beginning at $14.2 \mathrm{~Gy}$. Radiation portals were defined by means of 3-dimensional CT scan reconstruction and tailored to minimize toxicity to nearby structures, such as the brachial plexus, esophagus, and spinal cord.

\section{Surgical Approach}

Three to 4 weeks after completion of the induction therapy, patients were reassessed. Patients who did not show evidence of local disease progression or metastasis, as demonstrated on the basis of surgical lymph node restaging, underwent thoracotomy for resection of the primary tumor. Operative approaches included standard posterior-lateral, anterior-superior, and anterior hemiclamshell thoracotomies. The choice of specific surgical approach was dictated on the basis of the location and local invasiveness of the primary tumor. At the time of the operation, a lobectomy or pneumonectomy was performed. Lesser pulmonary resections were not used. Areas of direct tumor extension into the chest wall or spine were resected en bloc with the involved lung. Neurosurgical-orthopedic
TABLE 1. Patient demographics $(n=37)$

\begin{tabular}{lcc}
\hline & $\begin{array}{c}\text { No. of } \\
\text { patients }\end{array}$ & $\begin{array}{c}\text { Percentage of all } \\
\text { patients }\end{array}$ \\
\hline Sex & & \\
$\quad$ Male & 22 & 60 \\
$\quad$ Female & 15 & 40 \\
Preoperative stage NSCLC & & \\
IIB & 18 & 49 \\
IIIA & 8 & 22 \\
IIIB & 6 & 16 \\
IV (isolated brain metastasis) & 5 & 13 \\
Primary tumor histology & & \\
Squamous & 12 & 32 \\
Adenocarcinoma & 12 & 32 \\
Poorly differentiated & 13 & 35 \\
\hline
\end{tabular}

NSCLC, Non-small cell lung cancer.

consultation and operative assistance were used if spinal involvement required more than simple resection of the transverse process or wedge vertebral osteotomy. As needed, vertebrectomy was achieved posteriorly first, followed by standard en bloc resection of the entire specimen in continuity. Routine coverage of the bronchial stump with an intercostal or serratus muscle flap was used. Harvesting of the muscle flap was done on opening the chest. The choice of muscle flap used was dependent on the patient's anatomy and reach of the muscle flap, as well as surgeon preference. In general, the serratus muscle was used if the intercostal muscle bundle was insufficient or after pneumonectomy was performed. All visible and surgically accessible bronchopulmonary, hilar, and mediastinal lymph nodes were removed and submitted along with the primary lung resection specimen for pathology review. A pathologic complete response ( $\mathrm{p}-\mathrm{CR}$ ) was demonstrated when the resected surgical specimen contained no evidence of viable cancer. A resection was considered R0 when no viable tumor was left in the operative field, whereas an R1 resection left only microscopic tumor behind.

\section{Patient Follow-up}

After completion of multimodality treatment, all patients underwent close follow-up at our institution. Patients were evaluated every 3 months during the first 2 years postoperatively and then every 6 months thereafter on the basis of history, physical examination, and chest CT scan. Brain magnetic resonance imaging and CT scanning were done annually for the first 2 postoperative years or if symptoms indicated the need for more frequent scanning.

\section{Statistical Analyses}

Survival was determined by using Kaplan-Meier analysis. Log rank comparisons of survival were performed. Ninety-five percent confidence intervals (CIs) were calculated where appropriate. All patients are considered in the analysis of survival. Only patients who underwent resection are considered in the analysis of pathologic response. All data were analyzed with Graph-Pad statistical software (Graph Pad, Inc, San Diego, Calif). 
TABLE 2. Distribution of patients with $\mathrm{T} 3$ and $\mathrm{T} 4$ superior sulcus tumor

\begin{tabular}{lcc}
\hline TNM classification & No. of patients & Percentage of all patients \\
\hline Stage IIB & & \\
T3 N0 M0 & 18 & 49 \\
Stage IIIA & & 22 \\
T3 N2 M0 & 8 & 16 \\
Stage IIIB & & 2.7 \\
T3 N3 M0 & 1 & 11 \\
T4 N0 M0 & 4 & 2.7 \\
T4 N2 M0 & 1 & \\
Stage IV & & 14 \\
T3 N0 M1 (brain)* & 5 &
\end{tabular}

*All 5 patients presented with solitary brain metastasis and had negative mediastinal lymph nodes demonstrated by means of preresectional mediastinoscopy.

\section{Results}

\section{Patient Demographics}

From 1993 through 2003, 37 patients presented to the University of Maryland Thoracic Oncology Program and received multimodality treatment for superior sulcus lung cancers. The study population (Table 1) included 22 men (mean age, 55 years; range, 31-76 years) and 15 women (mean age, 56 years; range, 36-74 years). Primary tumor histology included squamous cell carcinoma $(\mathrm{n}=12)$, adenocarcinoma $(\mathrm{n}=12)$, and poorly differentiated carcinoma $(n=13)$. After mediastinoscopic staging of the mediastinum, preoperative TNM classification of the cohort revealed stage IIB $(\mathrm{n}=18)$, IIIA $(\mathrm{n}=8)$, IIIB $(\mathrm{n}=6)$, and IV $(\mathrm{n}=5)$ disease (Table 2).

Thirty-six of 37 patients underwent neoadjuvant chemoradiation, followed by surgical resection. Early in the series, one patient received preoperative high-dose radiation without chemotherapy, followed by surgical resection. Thirtyfour of 37 patients received platinum-based combination chemotherapy (carboplatin and paclitaxel, $\mathrm{n}=25$; cisplatin and etoposide, $\mathrm{n}=7$; or cisplatin and vinorelbine [Navel-

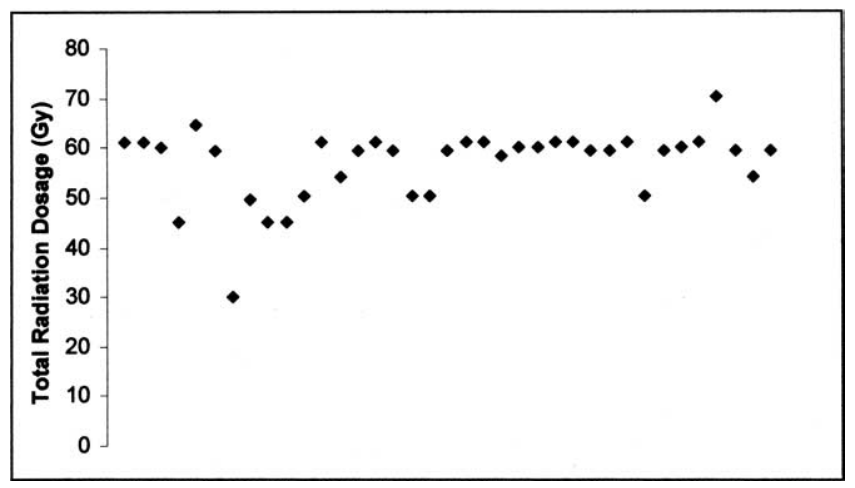

Figure 1. Distribution of total radiation dosages.
TABLE 3. Data on surgical resection

\begin{tabular}{lcc}
\hline & No. of patients & $\begin{array}{c}\text { Percentage of } \\
\text { all patients }\end{array}$ \\
\hline Operation type & & \\
$\quad$ Exploratory thoracotomy & 0 & 0 \\
$\quad$ Lobectomy & 34 & 92 \\
Pneumonectomy & 3 & 8 \\
Completeness of resection & & \\
$\quad$ Surgically complete (R0, R1) & 37 & 100 \\
$\quad$ Pathologically complete (R0) & 36 & 97 \\
\hline
\end{tabular}

bine, GlaxoSmithKline], $\mathrm{n}=2$ ). Two patients received single-agent neoadjuvant carboplatin in conjunction with high-dose radiotherapy. All but one patient completed the target high-level radiation dose. (The one exceptional patient did not progress past 30 Gy because of severe esophagitis, and this was experienced early in this series.) The mean total radiation dose for the cohort overall is $56.9 \mathrm{~Gy}$ (range, 30-70.2 Gy; Figure 1).

Surgical resection was feasible in all 37 patients and included 34 lobectomies and 3 pneumonectomies (Table 3 ). A small minority of tumors required pneumonectomy either because of large bulky disease that contiguously crossed the fissure $(\mathrm{n}=2)$ or because of central extension into the hilar region $(n=1)$. An R0 surgical resection was achieved in 36 (97.3\%) patients; the remaining patient had residual microscopic disease on the subclavian artery. This patient subsequently received subclavian stenting and additional focused external beam irradiation to this area. One elderly patient died in the perioperative period because of progressive sepsis and adult respiratory distress syndrome at approximately 30 days after surgical intervention, yielding an operative mortality rate of $2.7 \%(n=1 / 37)$. Significant morbidities occurred in 10 patients $(\mathrm{n}=10 / 37,27 \%$ patients) but were variable and without a dominant pattern (Table 4).

\section{Treatment Response and Survival Analysis}

A p-CR was found in 40.5\% (95\% CI, 26\%-59\%; $\mathrm{n}=15$ ) of patients. Mean follow-up for our entire cohort was 24.7

TABLE 4. Postoperative morbidity

\begin{tabular}{lcc}
\hline Morbidity & No. of patients & $\begin{array}{c}\text { Percentage of all } \\
\text { patients }\end{array}$ \\
\hline ARDS & 2 & 5.4 \\
Bronchopleural fistula & 2 & 5.4 \\
DVT & 2 & 5.4 \\
Prolonged atelectasis & 2 & 5.4 \\
Pulmonary embolus & 1 & 2.7 \\
Pneumonia & 1 & 2.7 \\
Hand paresthesia & 1 & 2.7 \\
Pleural effusion & 1 & 2.7
\end{tabular}

ARDS, Adult respiratory distress syndrome; DVT, deep venous thrombosis. 


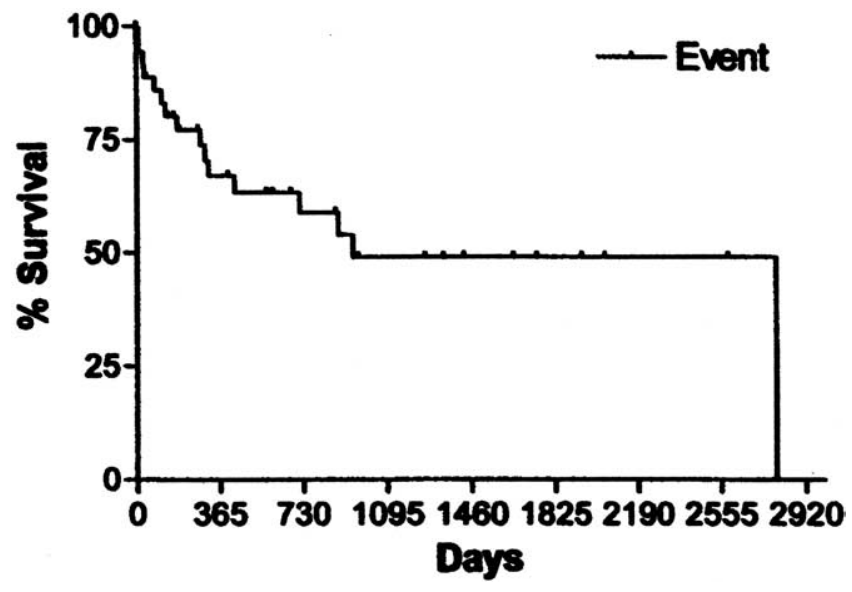

Figure 2. Overall survival of all eligible patients with follow-up.

months. The overall median survival time (MST) for the cohort available to follow-up $(\mathrm{n}=36)$ is 31.6 months or 2.6 years (Figure 2). The MST for patients with p-CR was 93.1 months or 7.8 years (Figure 3). The MST for patients with preresectional positive lymph nodes $(\mathrm{n}=12)$ remains undefined (MST not reached, Figure 4). The site of recurrence did not appear to influence survival (Figure 5). Statistically significant differences were not reached in survival comparisons involving $\mathrm{p}-\mathrm{CR}$, preresectional lymph node status, or pattern of recurrence.

\section{Recurrences and Disease Relapse}

Recurrences were found in 50\% (95\% CI, 33\%-67\%; $\mathrm{n}=18$ ) of all treated patients. Distant recurrences occurred in a total of 13 patients, and the most common site of distant metastasis was the brain $(\mathrm{n}=9,50 \%$ total number recurrences, $25 \%$ [95\% CI, 12\%-42\%] of all patients). The remaining distant

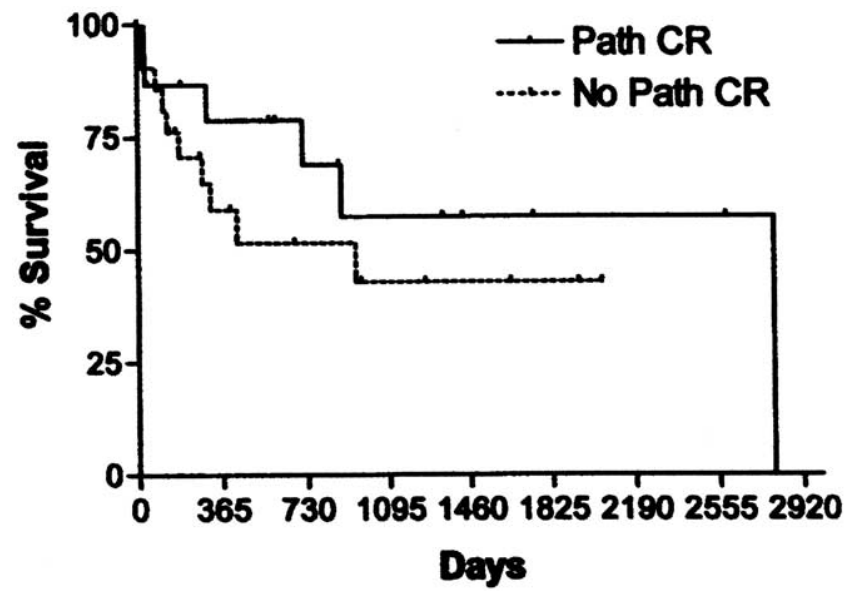

Figure 3. Overall survival of $\mathrm{p}$-CR versus no $\mathrm{p}$-CR $(P=.32)$.

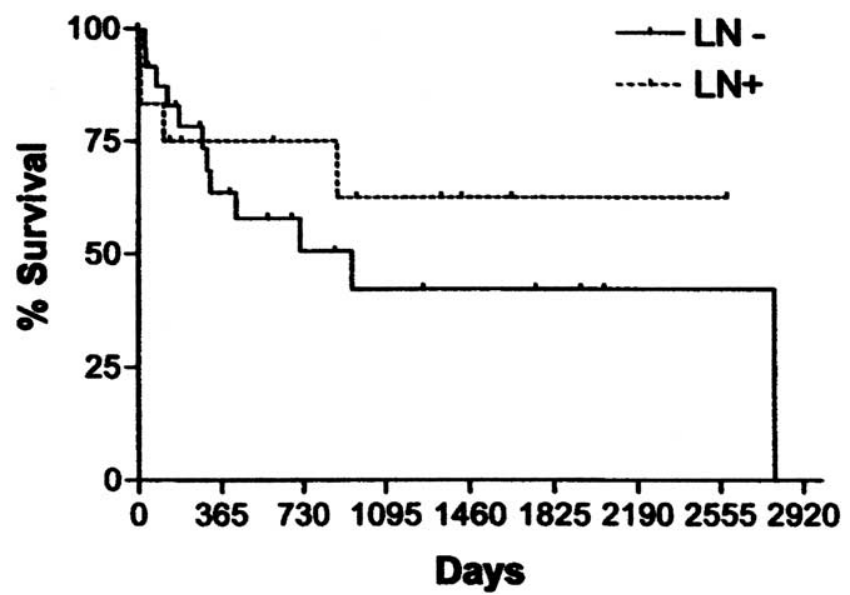

Figure 4. Survival by preresectional lymph node $(L N)$ status $(P=.49)$.

recurrences were accounted for by 4 patients (22.2\% total number recurrences, $11.1 \%$ [95\% CI, 3\%-26\%] of all patients) and included sites such as the adrenal, liver, and distant lymph nodes. Local recurrences, notably in the lung-mediastinum, occurred in 5 patients (27.7\% total recurrences, $13.8 \%$ [95\% CI, $5 \%-30 \%$ ] of all patients).

\section{Discussion}

Feasibility

One of the early challenges in treating Pancoast lung cancer centered on resectability. The addition of preoperative radiation, up to 30 to $35 \mathrm{~Gy}$, as initiated by Shaw and Paulson, did much to improve resectability rates but did not eliminate unresectability. Before the advent of 3-dimensional conformal radiotherapy, the total radiation dose that could be safely delivered was constrained by dose-limiting toxicities

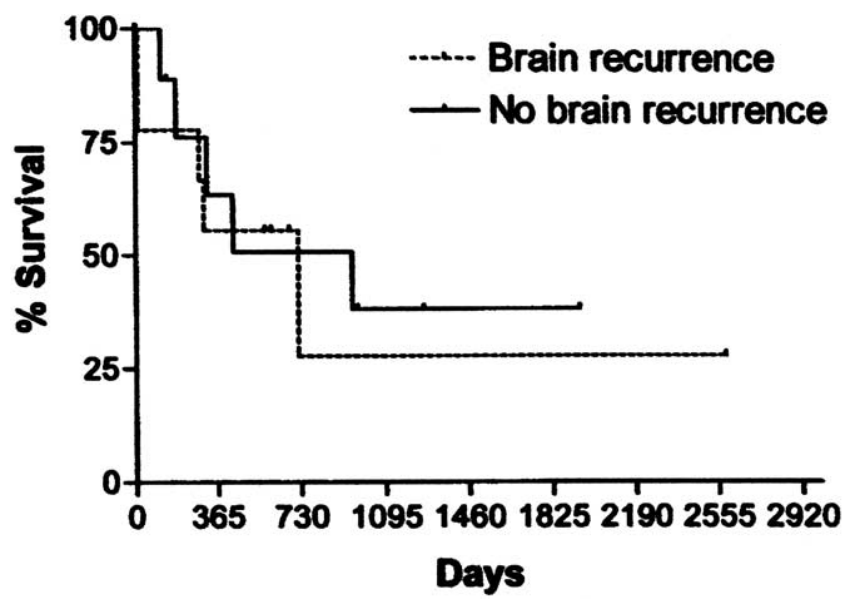

Figure 5. Recurrence pattern and survival $(P=.71)$. 
of nearby organs and anatomic structures in close proximity to Pancoast tumors, as well as impaired tissue healing postoperatively. Our present series demonstrates that highdose radiotherapy targeting to $60 \mathrm{~Gy}$ can be given in the neoadjuvant setting and is successfully tolerated in more than $97 \%$ of patients. Only 1 patient early in the series was unable to tolerate radiation past $30 \mathrm{~Gy}$ because of intractable radiation-induced esophagitis. The relative lack of significant radiation toxicity is directly attributable to the beneficial merits of precise 3-dimensional conformal radiation planning and the expertise of highly skilled radiation oncology collaborators. The potential gains achieved in local control from increased total radiation are tempered by the potential complications resulting from bronchopulmonary operations within an irradiated field. In our experience we have attempted to mitigate these circumstances at the time of the operation by avoiding unnecessary skeletonization and devascularization of the remnant bronchial stump. In addition, we have adopted the routine use of pedicled muscle flaps to provide healthy vascularized unirradiated tissue coverage to the bronchial stump. ${ }^{9}$ Although we experienced 2 postoperative bronchopleural fistulas in our series, the incidence of this dreaded complication was remarkably lower than would be otherwise anticipated given the high doses of radiation used.

\section{Surgical Outcomes}

Resectability was possible in all our patients, and an R0 resection was achieved in $97 \%$ of patients. This compares favorably with $\mathrm{R} 0$ resection rates seen in other contemporary series: Southwest Oncology Group (SWOG) ${ }^{6}$ 9416-45 Gy, T3 lesions (91\%) and T4 lesions (87\%); van Geel and colleagues ${ }^{10}-46$ Gy plus intraoperative radiation therapy (IORT) $10 \mathrm{~Gy}$ (48\%).

Our survival analysis also compares favorably with those from the SWOG 9416 trial. In SWOG 9416 T3 and T4 superior sulcus lungs with negative N2 lymph nodes were treated with neoadjuvant chemoradiation consisting of combination cisplatin and etoposide concurrent with 45 Gy of radiation. The 2 -year survival was $55 \%$ overall and $70 \%$ for those patients in whom R0 or R1 resection was attained. In our series we did not exclude patients with $\mathrm{N} 2$ involvement and administered platinum-based combination chemotherapy concurrent with average 56.9 Gy of radiation. We observed a 2-year survival rate of 59\%. The MST in our overall cohort was 2.6 years, and the MST was 7.8 years for patients with p-CRs. We also treated patients with Pancoast tumors with stage 4 disease, those presenting with solitary brain metastasis. Aggressive treatment of this notable subset of patients with stage 4 Pancoast disease appears justified in light of favorable survival data from patients with nonPancoast stage 4 disease with solitary brain metastasis in other series. ${ }^{11,12}$

\section{Mediastinal Lymph Nodes}

We did not exclude patients with Pancoast tumors with positive mediastinal lymph nodes from trimodality treatment. Contemporary with our series, SWOG 9416 excluded these patients from accrual, and we sought to offer treatment comparable with those who were not eligible for SWOG 9416. Interestingly, in our survival analysis the MST for patients with positive mediastinal lymph nodes was not reached, and moreover, the survival curves between positive and negative pretreatment mediastinal lymph nodes were not statistically different. We speculate that perhaps the inclusion of the mediastinum within the large-field irradiation portal might sterilize any tumor cells within the mediastinal lymphatics and therefore negates any potential clinical differences on the basis of nodal status. These results might suggest that pretreatment positive mediastinal lymph nodes should not necessarily preclude this subset of patients with Pancoast lung cancer from benefiting from trimodality treatment. However, there are few data to support proceeding with surgical resection in the face of persistent lymph node disease after completion of neoadjuvant therapy. The favorable prognosis for patients who experience mediastinal sterilization is consistent with the findings of other studies of multimodality therapy in stage III disease. ${ }^{13,14}$

\section{Role for Prophylactic Cranial Irradiation}

The most common site of metastasis was the brain, which accounted for $50 \%$ of all recurrences in our series and affected $25 \%$ of patients. These data corroborate results seen in other contemporary studies. ${ }^{6,15}$ Although Komaki and associates ${ }^{15}$ did not see a statistical difference in the frequency of brain metastasis between patients receiving versus not receiving prophylactic cranial irradiation (PCI), the group receiving PCI was disproportionately smaller in number than the non-PCI group. This issue will likely require a sufficiently powered study to determine the value of PCI. One ongoing trial, Radiation Therapy Oncology Group (RTOG) 0214, is a phase III study comparing PCI versus observation in patients with stage IIIA and IIIB non-small cell lung cancer. The target sample size is more than 1000 patients, which will provide sufficient statistical power to determine the efficacy of PCI. Because Pancoast tumors are relatively rare, it is not feasible to perform a similar PCI trial in only patients with Pancoast tumors. Therefore the outcome of RTOG 0214 might hold great interest for all who treat Pancoast tumors.

\section{Study Limitations}

The overall number of patients described in this series is based on one institution's experience. Despite inherent challenges with the coordination and execution of trimodality therapy, our study is nonetheless limited by its relative small size. This is especially unfortunate given the potentially 
favorable trends seen in $\mathrm{p}-\mathrm{CR}$ or in pretreatment lymph node status: increased statistical power might have resulted in statistically significant differences. Perhaps results such as ours from the present series, along with other contemporary experiences, might motivate consideration of a successor clinical trial to SWOG 9416, which might include patients with mediastinal lymph node involvement and perhaps consider inclusion of PCI.

\section{Summary}

Surgical resection of Pancoast tumors after neoadjuvant high-dose radiation therapy and chemotherapy can be safely performed, with improved clinical outcomes. High-dose radiation as part of a trimodality treatment regimen can be successfully tolerated and might confer a survival advantage. Pretreatment lymph node metastasis should not necessarily exclude patients from trimodality treatment. Local cancer control of Pancoast tumors can be accomplished with aggressive trimodality therapy, but the high number of distant metastases to the brain suggests that adjuvant PCI might be necessary to optimize long-term results.

\section{References}

1. Pancoast HK. Importance of careful roentgen-ray investigations of apical chest tumors. JAMA. 1924;83:1407-11.

2. Detterbeck FC. Changes in the treatment of Pancoast tumors. Ann Thorac Surg. 2003;75:1990-7.

3. Shaw RR, Paulson DL, Kee JL. Treatment of the superior sulcus tumor by irradiation followed by resection. Ann Surg. 1961;154:29-40.

4. Shaw RR. Pancoast's tumor. Ann Thorac Surg. 1984;37:343-5.

5. Martinez-Monge R, Herreros J, Aristu JJ, Aramendia JM, Azinovic I. Combined treatment in superior sulcus tumors. Am J Clin Oncol. 1994;17:317-22.

6. Rusch VW, Giroux DJ, Kraut MJ, Crowley J, Hazuka M, Johnson D, et al. Induction chemoradiation and surgical resection for non-small cell lung carcinomas of the superior sulcus: initial results of Southwest Oncology Group trial 9416 (Intergroup trial 0160). J Thorac Cardiovasc Surg. 2001;121:472-83.

7. Wright CD, Menard MT, Wain JC, Donahue DM, Grillo HC, Lynch $\mathrm{TJ}$, et al. Induction chemoradiation compared with induction radiation for lung cancer involving the superior sulcus. Ann Thorac Surg. 2002;73:1541-4.

8. Barnes JB, Johnson SB, Dahiya RS, Temes RT, Herman TS, Thomas CR. Concomitant weekly cisplatin and thoracic radiotherapy for Pancoast tumors of the lung. Am J Clin Oncol. 2002;25:90-2.

9. Sonett JR, Krasna MJ, Suntharalingam M, Schuetz J, Doyle LA, Lilenbaum R, et al. Safe pulmonary resection after chemotherapy and high-dose thoracic radiation. Ann Thorac Surg. 1999;68:316-20.

10. van Geel AN, Jansen PP, van Klaveren RJ, van Klaveren RJ, van der Sijp JRM. High relapse-free survival after preoperative and intraoperative radiotherapy and resection for sulcus superior tumors. Chest. 2003;124:1841-6.

11. Magilligan DJ Jr, Duvernoy C, Malik G, Lewis JW Jr, Knighton R, Ausman JI. Surgical approach to lung cancer with solitary cerebral metastasis: twenty-five years' experience. Ann Thorac Surg. 1986;42: 360-4.

12. Chidel MA, Suh JH, Greskovich JF, Kupelian PA, Barnett GH. Treatment outcome for patients with primary nonsmall-cell lung cancer and synchronous brain metastasis. Radiat Oncol Investig. 1999;7:313-9.

13. Albain KS, Rusch VW, Crowley JJ, Rice TW, Turisi AT, Weick JK, et al. Concurrent cisplatin/etoposide plus chest radiotherapy followed by surgery for stages IIIA (N2) and IIIB non-small-cell lung cancer: mature results of Southwest Oncology Group phase II study 8805 . J Clin Oncol. 1995;13:1880-92.

14. Turrisi AT, Scott CB, Rusch VR, Albain KS, Shepherd FA, Smith C, et al. Randomized trial of chemo-radiotherapy to $61 \mathrm{~Gy}$ [no S] versus chemoradiotherapy to 45 Gy followed by surgery [S] using cisplatin etoposide in stage IIIa non-small cell lung cancer (NSCLC): Intergroup trial 0139, RTOG (9309). Int J Radiat Oncol Biol Phys. 2003; 57(suppl):S125-6

15. Komaki R, Roth JA, Walsh GL, Putnam JB, Vaporciyan A, Lee JS, et al. Outcome predictors for 143 patients with superior sulcus tumors treated by multidisciplinary approach at the University of Texas M.D. Anderson Cancer Center. Int J Radiat Oncol Biol Phys. 2000;48:34754.

\section{Discussion}

Dr Joseph Miller (Atlanta, Ga). The historical evolution of successful treatment of Pancoast tumors dates from 1961, when the report by Shaw and Paulson was first presented. It is now 43 years later, and at the majority of the institutions, up until 2001, this bimodality therapy remained the predominant mode of treatment. In 2001, Valerie Rusch reported the results of the SWOG 9416 intergroup trial, which was the first time that combined chemotherapy and radiation therapy were done in a multi-institutional setting. This opened the way for the trimodality therapy with preoperative chemoradiation followed by surgical resection and became pretty much the standard way that this was done. Now the Maryland group has added high-dose 3-dimensional radiation with doses varying in their reported series from 5000 to $7000 \mathrm{~Gy}$, with the majority being at around 5700 to $5800 \mathrm{~Gy}$. The SWOG trial stopped at $4500 \mathrm{~Gy}$.

With this historical background, I have a few remarks and questions for the author. In their group they took on all comers. They had 18 patients with stage IIB, 8 patients with stage IIIA, 6 patients with stage IIIB, and 5 patients with stage IV disease. It is of note that in the article mediastinoscopy was performed in all patients before lymph node staging and before treatment. Complete resection was accomplished in an amazing number, $97.3 \%$, with a confirmed pathologic response, with 15 patients being $41.7 \%$. It is noted that they had a very high recurrence rate, higher than is reported in the majority of series, with a recurrence rate of 18 patients, with a rate of $50 \%$, the highest being noted by most centers always as being in the brain. Median survival for the entire group was 2.6 years, but for the complete pathologic response, it was 7.8 years. Perhaps one of the most interesting and yet to be determined points in the article is the following remark: "The median survival time of patients with positive preoperative lymph nodes remains undetermined because the number is not reached."

The authors state that pretreatment lymph node metastasis should not exclude patients from this modality. They also point out that with the high incidence of brain metastases, perhaps prophylactic cranial irradiation should be added to the standard armamentarium. The authors note that 3-dimensional radiation was given with $4500 \mathrm{~Gy}$ but with the additional boost of 14.2 Gy to the target area.

I would now like to ask the authors 3 questions. First, the authors reported using 3 different surgical approaches. Which one do they favor, and when is each indicated?

Dr Kwong. Thank you, Dr Miller, for your kind comments. 
In terms of surgical approaches, our most common approach is the posterior approach a la Paulson and Shaw. This approach is most common when we have the posterior associated Pancoast tumors without vessel or obvious brachial plexus involvement. Our second most common approach is the anterior Dartevelle approach, and that can be performed in a single stage or in 2 stages. For those apical tumors that are most anterior without posterior involvement, this can be performed in a single stage. For those that involve the posterior aspect of the chest wall, we do it in 2 stages, and that involves an anterior approach with a posterior approach. We find that the posterior approach adds a lot to the facility in terms of dissection in the hilum and the lymph nodes, at least in our experience. Lastly, the anterior clamshell approach, in which we perform a sternotomy and then tee it off in the neck along the border of the sternocleidomastoid, is very useful for those tumors that are abutting or potentially involve the great vessels, such as the innominate artery or the superior vena cava.

Dr Miller. My second question has 2 parts. The recurrence rate of $50 \%$ is higher than is reported in most series. How do the authors account for this? Is it because you are operating on all comers, including those with stage III and IV disease, and do you believe that this is justified?

Dr Kwong. Well, that is a very intriguing question. The recurrence of $50 \%$ is a little higher than that seen in other series, but we included patients who had a higher stage because of the mediastinal lymph node involvement, and that could perhaps play into the higher recurrence rate. The most common site of recurrence was the brain, and that is consistent with other series. As far as whether to include these patients, I think the telltale sign, although not definitive, is the breakdown slide of our median survival between those with positive and negative lymph nodes. I think the safe and only thing we can take away from that limited breakdown is that at least the group with positive lymph nodes did no worse than the group with negative lymph nodes, and therefore that seems to perhaps provide some degree of justification for inclusion.

Dr Miller. Finally, in the fact that essentially no patient was denied trimodality treatment, why not perform mediastinoscopy after the induction therapy with the chemotherapy and radiation and, if the nodes are positive, abort the procedure? I think this might decrease your high recurrence rate. I would appreciate your commenting on that, and I thank the association for the privilege of discussing this article.

Dr Kwong. Thank you, Dr Miller, for all of your questions.

The answer to that last question is, interestingly enough, that in all of our resection specimens, perhaps because of dumb luck, there was no lymph node involvement after treatment. Therefore that might not have impacted as well. However, your point is very important, and we now routinely do restaging mediastinoscopy, even after this type of induction treatment. If we were to encounter a patient who has mediastinal lymph node involvement at this stage, that patient would not be offered a surgical resection on the basis of the data we have across the literature today.

Dr Robert J. Cerfolio (Birmingham, Ala). Excellent work. I have a question and a comment, and it is very similar to Dr Miller's last question concerning when to do a mediastinoscopy. In my practice this is an example of a select group of patients that I do not do mediastinoscopy on if I suspect N2 disease: I will do it for suspected N3 disease but not for N2 disease. Unless you believe that repeat mediastinoscopy is safe and accurate after high-dose radiation, and I do not believe it is either, then why not save the mediastinoscopy for after the induction chemoradiotherapy. In general, because we all make some exceptions for the young healthy patients with recalcitrant N2 disease, do you offer resection for patients with recalcitrant $\mathrm{N} 2$ or N3 disease or do you deny resection and offer more chemotherapy, radiotherapy, or both?

Dr Kwong. Thank you very much, Dr Cerfolio.

I think I touched a little bit on that in the last question from Dr Miller. If the patient still presents with a recalcitrant lymph node that is not in direct juxtaposition to the primary tumor, those are patients who we would not go ahead and resect. However, if the lymph node is very close by, I think we sometimes reserve judgment to be very aggressive with these rare patients, and our current results might seem to provide a small justification for that.

Dr Cerfolio. Therefore, in selected patients with N2 disease, you would offer resection. A final important comment is that we are finding a survival advantage in our own data in patients who receive 60 Gy versus 45 Gy or less of preoperative radiation (not patients with Pancoast tumors but those with N2 disease), and I think we need to consider a prospective randomized trial looking at this question in an ACOSOG of some other type of multiinstitutional trial setting.

Dr Kwong. Thank you, Dr Cerfolio. I think that is a great last point. We can only speculate that perhaps our higher dose of radiation, especially when we have mediastinal involvement, that the large-field dose should encompass the mediastinum. In the SWOG study, N2 lymph nodes were excluded, and therefore there was no mediastinal sterilization with radiation. Perhaps mediastinal sterilization with our high-dose radiation might confer a survival advantage to that group equal to those who have negative lymph nodes at the time of preresection or pretreatment.

Dr Scott J. Swanson (New York, NY). Interesting and impressive series. I have one quick question. You only had one patient with subclavian vein involvement. Did you need to take vertebral body or the brachial plexus or any other structures in any of those cases given all the IIIB disease, and if you encounter positive margins, what is your intraoperative approach?

Dr Kwong. The answer is yes in terms of finding patients who have a small degree of involvement of the brachial plexus. If it is on the lower edge, we might go ahead and be a little bit aggressive. In terms of posterior vertebral involvement, we do see that, and actually a couple of our patients are included in that series. If it is just the transverse process or a partial osteotomy, we are very comfortable in performing that part of the procedure ourselves. However, if it is much more involved, where you have to take more of a hemivertebrectomy, then we involve neurosurgery or orthopedic consultation ahead of time and have them assist us in the operating room. In those cases they will go ahead and do the posterior aspect first and then we will go back and do the anterior aspect subsequently.

Dr Krasna. I thank everybody for their comments.

Dr Cerfolio hit on a couple of very good points. There is currently a new RTOG trimodality protocol. That protocol will have mediastinoscopy up front and then restaging, but actually, we excluded Pancoast tumors. Right now in our institution, in 
our new phase II study, we are doing just what you suggested, Dr Miller and Dr Cerfolio, and that is that we are waiting on the mediastinoscopy until after chemoradiation because of these results.

Lastly, Joe, just to comment, the other thing that you alluded to and that I do not want anybody to forget is that the morbidity of patients with Pancoast tumor without resection can be extremely high in terms of the local symptoms. I credit Joe McLaughlin for really pushing me in this area because one of the worst things is having a patient with a T4 Pancoast tumor who has received chemoradiation or radiation and you do not resect. Many of those patients go on with a horrible painful existence. Therefore we have become very aggressive also in terms of the local control to allow them their palliation.

\section{JTCVS On-Line Manuscript Submission and Review}

\section{Please visit http://www.editorialmanager.com/jtcvs/}

Effective September 15, 2001, authors and reviewers may submit manuscripts and reviews electronically via Editorial Manager, our new Web-based system with full electronic submission, review, and status update capabilities.

As we move from paper to electronic submissions, the Editorial Office will make proxy submissions of all manuscripts accompanied by a diskette containing the electronic files of the text, tables, and figures. Editors, authors, and reviewers will receive automatic e-mails when significant events occur.

We strongly encourage all authors and reviewers to use Editorial Manager. Although we will continue to accommodate the submission of paper manuscripts for some months, our goal is to be completely electronic within 9 to 12 months.

All individuals currently in our database for whom we have e-mail addresses will receive via e-mail a system-assigned username and password that can be used to log in to the system without prior registration. All those not receiving the e-mail must register the first time they use the system.

As with any broad systemic change, the conversion to the new system will take some time to complete. We ask your patience as we replace our in-office database with the new system. We also encourage you to take advantage of the speed and efficiency that the new system will provide for us all: editor, author, reviewer, and publisher. 